\title{
Partial graph design embeddings and related problems
}

\author{
Peter Jenkins
}

An embedding of a partial $G$-design $(X, P)$ is a $G$-design $(V, B)$ with the property that $X \subseteq V$ and $P \subseteq B$. In 1976, Wilson [4] showed, among other things, that any partial $G$-design can be finitely embedded; however, the order of Wilson's embedding is exponentially large with respect to the order of the partial design. The problem of finding small embeddings of partial $G$-designs for various graphs $G$ has received much attention in recent years, particularly for the case of partial Steiner triple systems and partial $m$-cycle systems with $m \geqslant 4$. The work of Andersen, Hilton, Mendelsohn, Hoffman, Lindner, Rodger, Bryant and others has seen considerable progress made on this problem. In contrast to this, the search for even a polynomial embedding of partial $(n, k, 1)$-BIBDs, for any given $k \geqslant 4$, has been unsuccessful. The closest result of interest to this problem is Hoffman and Lindner's $8 n+16 \sqrt{n}+82$ embedding for partial $\left(K_{4} \backslash K_{2}\right)$-designs [2]. While the graph $K_{4} \backslash K_{2}$ differs from $K_{4}$ (a block of size 4) by only one edge, Hoffman and Lindner's embedding relies heavily on the fact that $K_{4} \backslash K_{2}$ is tripartite. Since $K_{4}$ is not tripartite, a small embedding for partial $K_{4}$-designs (that is, partial $(n, 4,1)$ BIBDs) appears to be beyond the reach of the current methods.

In this thesis, we consider the problem of finding small embeddings of partial $G$ designs for various graphs $G$, as well as several closely related problems.

Given the attention the embedding problem has received for the case of cycles, the initial focus of this thesis is on constructing small embeddings for certain cycle-related graphs $G$. In Chapter 2, we consider the case when $G$ is either $D_{6}$ (a diagonal cycle of order 6 ) or a $(4,1)$-kite; note that both of these graphs are bipartite and differ from an even cycle by only one edge. Chapter 3 presents an embedding of partial $(m, k)$-kite designs for any given values of $m$ and $k$.

In Chapter 4, a technique is presented for obtaining a cubic embedding of a restricted class of partial $K_{4}$-designs. This embedding is valid for any partial $K_{4}$-design which has the property that every copy of $K_{4}$ contains at least two vertices which do not occur in any other copy of $K_{4}$.

\section{Received 1st December, 2005}

Thesis submitted to the University of Queensland, May 2005. Degree approved, November 2005. Supervisor: Associate Professor Elizabeth Billington.

Copyright Clearance Centre, Inc. Serial-fee code: 0004-9727/06 \$A2.00+0.00. 
The concept of irreducibility is introduced in Chapter 5, and is applied to 2-fold $m$ cycle systems. Specifically, it is shown that for any pair of integers $(m, n)$ with $4 \leqslant m \leqslant n$, if there exists an $m$-cycle system of order $n$, then there exists an irreducible 2-fold $m$ cycle system of order $n$, except when $(m, n)=(5,5)$. A similar result has already been established by Kramer [3] for the case of 3-cycles.

Chapter 6 describes further results on irreducible $\lambda$-fold $m$-cycle systems when $\lambda>2$. We prove the existence of irreducible 3-fold $m$-cycle systems of various orders, and show that for any $\lambda>2$, there exists an irreducible $\lambda$-fold $m$-cycle system of order $n$ for a sufficiently large integer $n$.

The problem of immersing partial Steiner triple systems is the focus of Chapter 7. An immersing of a partial Steiner triple system $(V, P)$ of order $n$ is a $\lambda$-fold triple system $(V, B)$ of order $n$ such that $P \subseteq B$. It is shown that a partial Steiner triple system of order $n$ can always be immersed in a 6-fold triple system of order $n$ if $n$ is odd, and a 12 -fold triple system of order $n$ if $n$ is even.

Finally, in Chapter 8 we turn our attention to pairs of orthogonal latin squares. Heinrich and Zhu [1] have shown that a pair of orthogonal latin squares of order $n$ can be embedded in a pair of orthogonal latin squares of order $t$ if $t \geqslant 3 n$, the bound of $3 n$ being best possible. Obtaining an analogous result for pairs of partial orthogonal latin squares has proved to be an extremely challenging problem. Although Lindner has proved that a pair of partial orthogonal latin squares can always be finitely embedded, there is no known method which obtains an embedding of polynomial order (with respect to the order of the partial arrays). The less difficult problem of embedding a single partial latin square in a pair of orthogonal latin squares is investigated in this chapter.

\section{REFERENCES}

[1] K. Heinrich and L. Zhu, 'Existence of orthogonal latin squares with aligned subsquares', Discrete Math. 59 (1986), 69-78.

[2] D.G. Hoffman and C.C. Lindner, 'A partial $K_{4} \backslash e$-design of order $n$ can be embedded in a $K_{4} \backslash e$-design of order at most $8 n+16 \sqrt{n}+82$ ', Australas. J. Combin. 29 (2004), 225-230.

[3] E.S. Kramer, 'Indecomposable triple systems', Discrete Math. 8 (1974), 173-180.

[4] R.M. Wilson, 'Decompositions of complete graphs into subgraphs isomorphic to a given graph', Congr. Numer. 15 (1976), 647-659.

Centre for Discrete Mathematics and Computing

Department of Mathematics

The University of Queensland

Queensiand 4072

Australia 